\title{
Computing Equilibrium in Matching Markets
}

SAEED ALAEI, Google Inc.

POOYA JALALY KHALILABADI, Cornell University

ÉVA TARDOS, Cornell University

\begin{abstract}
Market equilibria of matching markets offer an intuitive and fair solution for matching problems without money with agents who have preferences over the items. Such a matching market can be viewed as a variation of Fisher market, albeit with rather peculiar preferences of agents. These preferences can be described by piece-wise linear concave (PLC) functions, which however, are not separable (due to each agent only asking for one item), are not monotone, and do not satisfy the gross substitute property- increase in price of an item can result in increased demand for the item. Devanur and Kannan in FOCS 08 showed that market clearing prices can be found in polynomial time in markets with fixed number of items and general PLC preferences. They also consider Fischer markets with fixed number of agents (instead of fixed number of items), and give a polynomial time algorithm for this case if preferences are separable functions of the items, in addition to being PLC functions.

Our main result is a polynomial time algorithm for finding market clearing prices in matching markets with fixed number of different agent preferences, despite that the utility corresponding to matching markets is not separable. We also give a simpler algorithm for the case of matching markets with fixed number of different items.
\end{abstract}

CCS Concepts: •Theory of computation $\rightarrow$ Exact and approximate computation of equilibria;

\section{INTRODUCTION}

We consider the problem of matching without money with $n$ agents who have preferences over $m$ items. This problem models a range of situations from assigning students to schools, applicants to jobs, or people to committees. We call such an assignment problem a matching problem, if all agents are required to get a fixed number of items. An intuitive application is the school choice problem. Students have preferences over schools, and each student needs to get assigned to exactly one school. In this paper we will consider computing the fair randomized solution to this problem proposed by Hylland and Zeckhauser [12] based on market equilibria.

We model the preferences of agents with the value of agent $i$ for being assigned to item $j$ is $v_{i j}$. Using values allows agents to express the intensity of their preferences. An important property of an allocation is its efficiency. Since agents utilities are meaningless to compare (without money, there is no natural unit to express utility), the best we can hope for is a Pareto-efficient allocation. An allocation is Pareto inefficient if there is an alternate allocation where no agent is worse off, and at least one agent has improved utility. We will consider fractional or randomized allocation. The

Permission to make digital or hard copies of all or part of this work for personal or classroom use is granted without fee provided that copies are not made or distributed for profit or commercial advantage and that copies bear this notice and the full citation on the first page. Copyrights for components of this work owned by others than the author(s) must be honored. Abstracting with credit is permitted. To copy otherwise, or republish, to post on servers or to redistribute to lists, requires prior specific permission and/or a fee. Request permissions from permissions@acm.org.

EC17, June 26-30, 2017, Cambridge, MA, USA.

ACM ISBN 978-1-4503-4527-9/17/06 ... \$15.00

http://dx.doi.org/10.1145/3033274.3085150

(c) 2017 Copyright held by the owner/author(s). Publication rights licensed to ACM. 
value of an agent $i$ for the fractional allocation $x_{i j}$ is $\sum_{j} v_{i j} x_{i j}$, if it obeys the matching constraint. This is the agent's expected value, if $x_{i j}$ is the probability of assigning item $j$ to agent $i$.

Market equilibria offer an intuitive, fair, and Pareto-efficient solution for problems of allocations of resources to agents who have their own (incomparable) preferences over the items in systems with no money. This was proposed by Hylland and Zeckhauser [12] in the context of matching markets, and by Dolev et al. [9] (see also [11] and [13]), in the context of allocation of resources in systems. The idea is to endow each agent with equal resource: a unit of (artificial) money. A set of prices $\mathbf{p}$ for the items is market clearing, if there is a fractional allocation $\mathbf{x}$ of items to agents such that the following conditions hold (i) each item is allocated at most once, (ii) each agents is allocated her favorite set of items subject to the budget constraint ${ }^{1}$ that $\sum_{j} x_{i j} p_{j} \leq 1$, and (iii) the market clears, meaning that all items not fully allocated have price 0 . Hylland and Zeckhauser [12] showed that such market equilibria is guaranteed to exists, see also Appendix $C$ of the full version of our paper Alaei et al. [3]. We view the resulting fractional (or randomized) allocation $\mathrm{x}$ as a fair solution to the allocation problem without money, which is also clearly Pareto-efficient and envy-free (no agent prefers the allocation of another agent). ${ }^{2}$ We are concerned with computing this solution efficiently.

Computing Market Equilibria and the Odd Demand Structure of Matching Markets. Market equilibrium problems where demands satisfy the gross substitute condition are well understood [7], and can be computed efficiently. The demand structure of our matching problem does not satisfy the gross substitutability condition, which requires that decreasing the price of an item (while keeping all other prices fixed) should never decrease the demand for that item. We show an example in Appendix B of the full version of this paper Alaei et al. [3] that decreased price can cause decreased demand in a matching markets. It is not hard to gain intuition for the phenomena: with the decreased price the agent could get her old allocation, and would have money leftover. In other markets, money can be used to buy additional items. However, in a matching market additional money makes no sense, and instead, the agent may want to exchange his share of a cheaper and less favorable items (possibly the item whose price decreased) for share of a more valuable expensive items.

Devanur and Kannan [8] gave an algorithm to compute market equilibria in markets with a fixed number of items, where agents have piece-wise linear concave (PLC) utility functions, despite the fact that PLC utility functions can give rise to demand not satisfying the gross substitute condition. They also gave a polynomial time algorithm to compute market equilibria in markets with a fixed number of agents, where agents have piece-wise linear concave and separable utility functions. They leave as an open problem to give a polynomial time algorithm to compute market equilibria in markets with a fixed number of agents and general PLC utilities that are not separable. We will show in Section 2 that demand structure of the matching market can be modeled by a piece-wise linear concave (PLC) utility function, which however, is neither separable nor monotone. This allows us to use the algorithm of [8] to find a market equilibrium is the number of goods is fixed, but leaves open the question whether market equilibrium can be found in polynomial time if the number of different agents is finite instead.

Our Results. We give a polynomial time exact algorithm for finding market equilibria of matching markets with a fixed number of agents, extending the work of [8] to the case of matching markets with a fixed number of agents, despite the fact that utilities describing matching markets are not separable. Our algorithm in Section 3 is based on the structural Theorem 3.10, and explores a polynomial number of possible player utility values and allocation structures,

\footnotetext{
${ }^{1}$ Note that agents have no use for the (artificial) money and are simply optimizing their allocated item, subject to their budget.

${ }^{2}$ An alternate way to arrive to the same solution concept is to assign each agent an equal share of each resource, and then look for an equilibrium of the resulting exchange market. To see that this results in an identical outcome, we can think of each agents trade, as a two step-process, where he first sells all his allocated share on the market prices, and then uses the resulting money to buy his optimal allocation.
} 
and finds a market equilibrium in polynomial time when the number of agents is fixed. The algorithm also extend to the case when there are only a finite number of different agent utility types.

In case of large number of items and finite number of agents, when each individual item is insignificant, our allocation can be used for finding an approximately optimal integer solution. We achieve this by showing in Lemma 3.8 that we find an allocation in which the number of items which are shared by the agents is $O\left(n^{2}\right)$, which is constant when the number of agents is constant.

In Appendix A of the full version of this paper Alaei et al. [3], we consider the problem with a fixed number of goods. In this case, the algorithm of [8] can find market equilibria in polynomial time. We give a simpler algorithm which is tailored for matching markets. With $m$ different goods and $n$ agents, our algorithm enumerates a polynomial number of different set of prices and allocation structures for the equilibrium.

Related Work. The problem of fairly allocating items to unit demand agents without money has been studied extensively in both Economics and Computer Science literature. Perhaps the most well known solution to this problem is the random serial dictatorship (RSD) [1] - also known as random priority $(R P)$ - in which agents are served sequentially according to a random permutation, and each agent in turn receives her most preferred item among the remaining ones. Clearly, serial dictatorship is Pareto efficient, and as a result, RSD is ex post Pareto-efficient, i.e., Pareto-efficient given the order used. However, the expected allocation of RSD may not be Pareto-efficient, i.e., its not interim Pareto-efficient. In Appendix B of the full version of this paper [3], we give an example where the expected allocation of RSD can be Pareto improved just using the order of player preferences, showing that RSD may be Pareto-inefficient even with ordinal preferences.

An alternative solution called probabilistic serial (PS) was proposed by Bogomolnaia and Moulin [6] which is both envy-free and Pareto-efficient with respect to ordinal preferences. The PS mechanism is, however, not Pareto-efficient with cardinal preferences. This is possible, as ordinal preferences are not always sufficient for ranking the randomized (interim) allocations, (i.e., ranking of distributions over outcomes).

The mechanism we study in this paper, based on market equilibrium from equal income, has been proposed in this context by Hylland and Zeckhauser [12], is both envy-free and Pareto-efficient even with respect to cardinal preferences. Note that neither PS nor the market equilibrium mechanism is strategy-proof. However, Zhou [14] shows that for $n \geq 3$ agents there is no mechanism that is strategyproof, Pareto-efficient, and envy-free.

Hylland and Zeckhauser [12] proves that equilibrium is guaranteed to exist (see also Appendix C of the full version of our paper Alaei et al. [3], and propose an exponential time algorithm for finding approximate equilibrium, whereas the current paper proposes an exact algorithm for computing equilibrium which runs in polynomial time when either the number of agents or the number of items is constant. Most of the recent work on the problem of assignment without money has been focused on analyzing the efficiency of RSD and PS mechanism under cardinal and/or ordinal preferences, e.g., Adamczyk et al. [2], Bhalgat et al. [5].

The main techniques used in the current paper are based on the cell decomposition result of Basu et al. [4] which has also been used by Devanur and Kannan [8] to derive a polynomial time algorithm for a related market equilibrium computation problem. We show how to find equilibria of matching problems in polynomial time when the number of agents is fixed. In Appendix A of the full version of this paper [3], we also give an algorithm to find equilibria in matching markets with a fixed number of goods. While the algorithm of [8] can be used for this latter case, our algorithm is simpler: we avoid some complications (for instance their primal dual technique for checking the market clearing conditions), and we use a simpler cell decomposition theorem. The case of fixed number of agents has been 
studied by [10]. However, they assume that the agents' utility functions are strictly concave and strictly monotone, which does not apply to our problem. They also approximate the Walrasian equilibrium, while our main goal is to find the exact value of equilibrium prices and allocations.

\section{PRELIMINARIES}

In this section we review the matching problem with additive preferences and the market equilibrium solution we aim to compute. Then we'll discuss our main technical tool, the cell decomposition technique of Basu, Pollack, and Roy [4].

The Matching Problem. The problem is defined by a set of $m$ items, and $C_{j} \geq 0$ amount available of item $j$, and a set of $n$ agents. The matching problem requires that we allocate exactly 1 unit of these items to all agents. The amount $C_{j}$ available of each item $j$ may be very small, so the 1 unit allocated to an agent may need to be combined from small fractions of many different items. An allocation $\left\{x_{i j}\right\}$ for all agents $i$ is a feasible solution of the matching problem, if

$$
\begin{aligned}
\sum_{j} x_{i j} & =1 \text { for all } i \in[n] \\
\sum_{i} x_{i j} & \leq C_{j} \text { for all } j \in[m] \\
x_{i j} & \geq 0 \text { for all } i \in[n] \text { and } j \in[m]
\end{aligned}
$$

We assume agent $i$ has value $v_{i j}$ for a unit of item $j$. So her value for a set of $\left\{x_{i j}\right\}$ amounts of each item $j$ is $\sum_{j} v_{i j} x_{i j}$, assuming $\sum_{j} x_{i j}=1$.

More generally, we can require to allocate different amounts for different agents, and allow the matching constraint to be only an upper bound, that is, allocate at most 1 unit to each agent. For simplicity of presentation, in this paper we will use equal amount required for the agents, and normalize that value to 1 . Further, we will assume, also for simplicity of the presentation only, that $\sum_{j} C_{j}=n$, so a feasible solution to the matching problem will fully allocate all items.

Fair Allocation: Matching Market. We use the Fisher market proposed by Hylland and Zeckhauser [12] to make the allocation fair. Fisher market is defined by giving each agent a unit of (artificial) money. A market equilibrium is defined by a set of prices $p_{j} \in \mathbb{R}_{\geq 0}$ for each item $j$. Given a set of prices, the agent $i$ 's optimization problem can be written as follows:

$$
\begin{gathered}
\sum_{j} v_{i j} x_{i j} \\
\sum_{j} x_{i j}=1 \\
\sum_{j} x_{i j} p_{j} \leq 1 \\
\text { subject to } \\
x_{i j} \geq 0
\end{gathered} \quad \text { (The matching constraint) }
$$

A market equilibrium for this market is a set of prices $\mathbf{p}$, and a feasible allocation $\mathbf{x}$ such that (i) $\mathbf{x}_{i}$ is an optimal solution to agent $i$ 's optimization problem with respect to prices $\mathbf{p}$ and her budget of 1 unit of money. Note that the requirement of market clearing that all items are allocated, is automatically satisfied due to the matching constraint and our assumption that $\sum_{j} C_{j}=n$. 
The matching constraint in the agents' preferences creates an odd demand structure. For some prices the agent's optimization problem is not feasible, and even on prices when all agent optimization problems are feasible, the preferences do not satisfy the gross substitute property, i.e increasing price of an item may increase the demand of that item, as explained in the introduction, and an example is shown in Appendix B of the full version of this paper [3].

A natural idea to convert the problem to one with a simpler structure is to allow agents to have free disposal, i.e., if assigned more than 1 unit of item, they value the best unit. Unfortunately, this change in the model significantly changes the structure of the problem, and can result in market equilibria that are simply not feasible for the original problem. Consider the following market with 2 agents and 2 items.

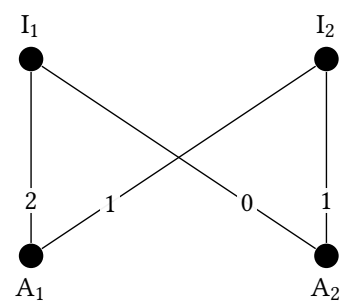

Let $v_{1}=(2,1)$ and $v_{2}=(0,1)$. Assume that the prices are $\mathbf{p}=(0.5,1.5)$. When agent's have free disposal, these prices are equilibrium, since $\mathbf{x}_{1}=\left(1, \frac{1}{3}\right)$ and $x_{2}=\left(0, \frac{2}{3}\right)$ is optimal for both agents, and also clears the market. However, with matching market preferences these prices are not equilibrium, since $A_{1}$ will not get any of $I_{2}$ and $A_{2}$ cannot afford all of $I_{2}$ so the prices are not market clearing.

We note that it is possible to express this matching market problem as a classical Fisher market with agent preferences that are piece-wise linear and concave (general PLC) functions, though non-monotone and non-separable. To do this, we first relax the matching constraint in the agents' optimization problem to requiring only that $\sum_{j} x_{i j} \leq 1$. We will show that the market clearing condition of a Fisher market requiring that all items are allocated (or have 0 price) will help to enforce that all agents get exactly 1 unit. We can also express the agents' utility as a piece-wise linear and concave function. To do this, let $v_{i}^{*}=\max _{j} v_{i j}+\epsilon$ where $\epsilon>0$. Let agent $i$ 's utility for an allocation $\mathbf{x}_{i}$

$$
\min \left(\sum_{j} v_{i j} x_{i j}, \sum_{j} v_{i j} x_{i j}+v_{i}^{*}\left(1-\sum_{j} x_{i j}\right)\right) .
$$

When $\sum_{j} x_{i j} \leq 1$, the first term is smaller, but when $\sum_{j} x_{i j} \geq 1$ the minimum is taken by the second term, so the total value strictly decreases as the allocated amount exceeds 1 . Since the agent's utility decreases with more than one unit allocated, an optimal solution to the agent's optimization problem will allocate at most 1 unit of item to each agent, and hence if the market clears, this is also a solution to the matching market problem.

Devanur and Kannan [8] provides a polynomial time algorithm to find the equilibrium prices and allocation for the case with fixed number of goods. In Appendix A of the full version of this paper [3], we give a simpler exact algorithm for this case, taking advantage of the matching structure. Our main result is to extend this to the case of fixed number of agents, instead of fixed number of items. [8] also offers an algorithm for finding market clearing prices for with fixed number of agents, but only with separable PLC utilities, i.e., when the utility of each agent is a separable function of the items allocated to the agents. Note that the utility function of a matching problem is necessarily not separable, as it needs to express the upper bound on the total allocation.

The Cell Decomposition Technique. Our algorithms will search the space of optimal utility values of each agent, and for each possible utility value, will search through the possible structures of allocations. There are only a fixed number of 
agents, however, the space of possible optimal values is huge. We use a cell decomposition technique to make the search space discrete, facilitated by a characterization of equilibria. The beginning of Section 3 has a more detailed outline.

A main technical tool for our work will be the following theorem concerning the way polynomials divide the space in a $d$ dimensional space. Given a set of polynomials on $M$ variables, the sign of the polynomials define an equivalence between vectors in the $M$ dimensional space $\mathbb{R}^{M}$, where two vectors $y$ and $y^{\prime}$ are equivalent if all polynomials have the same sign on $y$ and $y^{\prime}$. We call the equivalence sets of this relations the cells of the way the polynomials divide up the space. In principle $N$ functions can divide up the space into at many as $3^{N}$ cells (as each polynomial can be 0 positive or negative). However, Basu, Pollack, and Roy [4] showed that bounded degree polynomials in small dimensional spaces define much fewer cells.

THEOREM 2.1. [4] If we have a set of $M$ number of variables, and $N$ number of polynomials whose degree is at most $d$, then the number of non-empty cells, and the time required to enumerate them is $O\left(N^{M+1}\right) d^{O(M)}$.

We will use this decomposition to find the equilibrium for our matching problem. To illustrate the idea, and let $\mathbb{R}^{n}$ be the space of all possible agent utilities. Roughly speaking the idea is as follows. If we could describe whether a set of utilities $u \in \mathbb{R}^{n}$ arises from an equilibrium by the signs of a few bounded degree polynomials in these variables, then we could use Theorem 2.1 to enumerate all cells defined by these polynomials, and test which of the cells satisfies the condition required for being an equilibrium. Unfortunately, the equilibrium condition cannot be described this way, so we will need to introduce additional variables (helping us infer the prices and assignment, despite the fact that these are not in fixed dimensional space) to be able to carry out this plan.

\section{COMPUTING MARKET EQUILIBRIUM WITH FIXED NUMBER OF AGENTS}

In this section, we give an exact algorithm to find an equilibrium in the case where the number of agents $n$ is constant, and the number of different goods is an arbitrary number $m \in \mathbb{N}$, under the mild technical assumption that each agent has a unique most preferred item. More formally, for every agent $i \in[n]$, there is exactly one item $j$ such that $v_{i j}=\max _{k \in[m]}\left(v_{i k}\right)$. The goal of this section is proving the following theorem.

THEOREM 3.1. Exact equilibrium (prices and allocations) in a matching market with fixed number of agents, in which agents have additive values one unit of money, and a unique most preferred item, can be found in polynomial time.

General Outline and Techniques. Our algorithm searches the space of agents' optimal utilities and item prices to find an equilibrium. We divide this space into a polynomial number of cells, where each cell contains utility and price vectors that have the same properties. We use Theorem 2.1 as the basis of the cell decomposition. The space of possible agent utilities is finite dimensional. However, since the number of items is not constant, we cannot use a separate variable for each item price. In section 3.1, we provide a bundling technique and a characterization of the equilibrium structure that allow us to define equilibria using only a finite set of variables.

Cell. Consider the vector of player utilities $\mathbf{u}=\left\{u_{i}\right\}$, a constant dimensional space for fixed number of agents. Now consider the linear functions $u_{i}-v_{i j}$ for each agent $i$ and item $j$. A cell of the space of utilities $\mathbf{u}$ defined by these functions is the region of this space in which each of these functions has a fixed sign. Within each region, the items are divided for each agent $i$ into those with value above $u_{i}$, same as $u_{i}$, and below $u_{i}$. This division also has implications on prices: if the utilities are part of an equilibrium, the price $p_{j}$ of any item $j$ with value $v_{i j}>u_{i}$ will have to be above 1 . We will add further variables and polynomials, until each cell provides enough information for checking all the equilibrium properties. 
Layered Cell Decomposition. Next we would like to add the item prices as variables. However, to keep the running time polynomial when using Theorem 2.1 , we can only have a constant number of variables, so we cannot afford a variable for each item price. To get around this problem we will try a polynomial number of different structures for the price vector, where for any fixed structure, we can define all item prices via a fixed number of variables. To do this, for each agent we will fix a special item that is at least partially allocated to the agent. Lemma 3.6 will show that given prices for the fixed number of special items, we can infer prices for all items.

Finally, we also need to be able to find the assignment variables. We will show in Lemma 3.8 that each pair of agents only shares a few items (at most 5), and given the set of shared items, as well as the utilities and item prices, the allocation can be fully determined. Our algorithms iterates over all structures of specially assigned items and shared sets of items. For each of these structures, the algorithm iterates over all cells of the cell-decomposition given by agent utilities and prices of special items and the constraint (polynomials) described in the next subsections that ensure that these describe an equilibrium, and finds the ones which correspond to equilibria. ${ }^{3}$

Bundles. Rather than thinking about individual items in isolation, it is useful to think of items in pairs. In equilibria each agent spends exactly one unit of money and gets exactly a total of one unit of items. This means that in equilibrium, if an agent gets some amount an item whose unit price is less than one, she should also get some amount of an item whose unit price is more than one. As suggested by this fact, we pair items of price below and above 1 . We define a bundle as either a single item of price 1, or fractions of two items of a total of one unit of items, where the total unit price of the bundle is exactly 1 . First, we show that in a market equilibrium, the allocations of items to agents, can be rewritten as the allocation of bundles to agents.

Lemma 3.2. In an equilibrium pricing $\mathbf{p}$ with equilibrium allocation $\mathbf{x}$, there exists a bundling $B$ of items such that

a. Each bundle $b$ consists of at most two items. Two items $j$ and $k$ are in a bundle $b=(j, k)$ if and only if $p_{j}<1<p_{k}$. One item $j$ forms a bundle $b=(j)$ if and only if $p_{j}=1$.

b. Each bundle $b=(j, k)$, associated with a unique mix $0<\alpha_{b}<1$ such that $\alpha_{b} p_{j}+\left(1-\alpha_{b}\right) p_{k}=1$ (recall that $\left.p_{j}<1<p_{k}\right)$. For bundles with one item we use $\alpha_{b}=1$.

c. The optimum allocation of items to agent $i$ satisfying the matching constraint can be rewritten as allocation of bundles.

Proof. Let $\mathbf{p}$ be an equilibrium pricing and $\mathbf{x}$ be an arbitrary allocation associated with $\mathbf{p}$. For each agent $i$, we know that the total allocation of items to $i$ and the the total cost of $i$ 's items is 1 (due to the market clearing condition), i.e.

$$
\sum_{j \in[m]} x_{i j}=1
$$

and

$$
\sum_{j \in[m]} p_{j} x_{i j}=1
$$

We start rewriting $i$ 's allocation with bundles. Let $y_{i b}$ be the amount of bundle $b$ that $i$ uses in the new allocation $\left(y_{i b}\right.$ is zero at the beginning). At each step we consider the following cases

(1) For every $j$ that $x_{i j}>0$, we have $p_{j}=1$ : in this case all such items should be in a bundle $b=(j)\left(y_{i b}=x_{i j}\right)$, so our claim is correct.

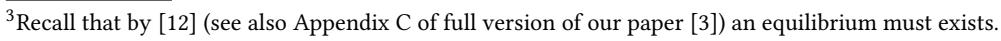


(2) There exists $j$ that $x_{i j}>0$ and $p_{j}>1$ : This means that there exists another item $j^{\prime}$ such that $p_{j^{\prime}}<1$ and $x_{i j}>0$, otherwise if $i$ gets $t$ unit of items, her total cost cannot be $t$ (note that $t=1$ here). So by the definition, $j$ and $j^{\prime}$ should be in a bundle $b=\left(j, j^{\prime}\right)$.

Let $z=\min \left(\frac{x_{i j}}{\alpha_{b}}, \frac{x_{i j^{\prime}}}{1-\alpha_{b}}\right)$. In the second case, we increase $y_{i b}$ by $z$, and reduce $x_{i j}$ by $z \alpha_{b}$ and $x_{i j^{\prime}}$ by $z\left(1-\alpha_{b}\right)$. This means that both the total cost of remaining allocation of $\mathbf{x}$ and the total remaining items in allocation of $\mathbf{x}$ decrease by $z$. Note that by doing this the total allocation of $i$ (counting her allocation in $\mathbf{x}_{i}$ and $y_{i}$ ) does not change. Furthermore, either $x_{i j}$ or $x_{i j}$ becomes zero.

If we repeat this process, $y_{i}$ gives us a way to rewrite the allocation of items to $i$ as an allocation of some bundles $\left(B_{i}\right)$ to $i$. If we repeat this for all the agents, we get what we want.

For each agent $i$, the value of a bundle $b=(j, k)$ is $v_{i b}=\alpha_{b} v_{i j}+\left(1-\alpha_{b}\right) v_{i k}$, while the value of a single item bundle $b=(j)$ is just the value $v_{i b}=v_{i j}$. Let $B_{i} \subseteq B$ the set of bundles of maximum value for agent $i$ (called $i$ 's optimum bundles).

COROLlary 3.3. The optimum allocation of items to agent $i$ is any allocation of bundles using only bundles in $B_{i}$. Furthermore, utility of $i$ in the equilibrium is $u_{i}=v_{i b}$ for the bundles $b \in B_{i}$.

Proof. We prove this by contradiction. Assume that $\mathbf{y}$ is the allocation of bundles to agents. Also assume that there exists an agent $i$ and a bundle $b$ for which $y_{i b}>0$ but there exists another bundle $b^{\prime}$ such that $v_{i b}<v_{i b^{\prime}}$. Since the unit price of the bundles is 1 , and there is one unit of items in them, $i$ can trade her share of $b$ for the same amount of $b^{\prime}$ and increase her value. This is a contradiction.

Now since the unit values of bundles that $i$ uses are the same, and $i$ gets exactly one unit of bundles, her utility in equilibrium is equal to the unit value of these bundles. So the second claim is also true.

A key observation for using the bundles to define prices in Lemma 3.6 is the following exchange property of optimal bundles.

LemmA 3.4. If $b=(j, k)$ and $b^{\prime}=\left(j^{\prime}, k^{\prime}\right)$ are optimum bundles of agent $i$ (are in $\left.B_{i}\right)$, such that $p_{j}, p_{j^{\prime}}<1<p_{k}, p_{k^{\prime}}$, then $b^{\prime \prime}=\left(j, k^{\prime}\right)$ and $b^{\prime \prime \prime}=\left(j^{\prime}, k\right)$ are also in $B_{i}$.

Proof. We prove this lemma by using contradiction. W.l.o.g assume that $b^{\prime \prime} \notin B_{i}$. If in equilibrium $i$ trade her bundles to get $y_{i b}>0$ unit of $b$ and $y_{i b^{\prime}}>0$ unit of $b^{\prime}$, then by lemma 3.3, her value will not change. Now, since $x_{i j}>0$ and $x_{i k^{\prime}}>0$, similar to proof of lemma 3.2, we can rewrite her allocation so that it includes some of $b^{\prime \prime}$, and similarly rewrite the remaining allocation of $i$ with other bundles. Since the unit value of $i$ for $b^{\prime \prime}$ is less than value of $i$ in equilibrium, this is a contradiction with corollary 3.3 and we are done.

\subsection{Characterizing the Prices and Optimum Bundles with Polynomials}

In this subsection we define a set of variables and polynomials that help us determine agent utilities and prices of all items at equilibrium. We consider assignments in the next subsection.

For each agent $i$, we define a variable $u_{i}$ which is $i$ 's utility in the equilibrium. By Lemma 3.2, we know that any equilibrium allocates bundles to agents, where each agent only gets one unit of her optimum bundles $B_{i}$. Since we did not define variables for the prices yet, we cannot use prices to define bundles, so we start by defining a set of item bundles for each agent just based on the fact from Corollary 3.3 that optimum bundles must give value $u_{i}$. 
Candidate Bundles. For each agent $i$, we define a candidate bundle to be the items whose value is $u_{i}$, or the pair of items $j$ and $k$ such that $v_{i j}<u_{i}<v_{i k}$, so there exists a unique $0<\alpha_{j k}^{i}<1$ such that $\alpha_{j k}^{i} v_{i j}+\left(1-\alpha_{j k}^{i}\right) v_{i k}=u_{i}$. Note that the optimum bundles of $i$ also satisfy this constraint (by Corollary 3.3). This means that the optimum bundles of an agent is a subset of her candidate bundles. In addition, the price of optimum bundles is exactly 1.

In order to find the set of candidate bundles of agent $i$, we define a polynomial $v_{i j}-u_{i}$ for each agent $i$ and item $j$. This way, each cell tells us for each item $j$, whether $v_{i j}<u_{i}, v_{i j}=u_{i}$ or $v_{i j}>u_{i}$. For any two items $j, k \in[m], j$ and $k$ form a candidate bundle if $v_{i j}<u_{i}$ and $v_{i k}>u_{i}$. Similarly if for an item $j$, if $u_{i}=v_{i j}$ then $j$ alone forms a candidate bundle. By the information provided by each cell, for each agent $i \in[n]$ and item $j, k \in[m]$ that form a candidate bundle, we define the ratio for the candidate bundle to be $\alpha_{j k}^{i}=\frac{u_{i}-u_{k}}{u_{j}-u_{k}}$.

Not all the candidate bundles of agent $i$ are in the set of her optimum bundles, since the price of optimum bundles should be exactly 1 . We first observe that the unit price of a candidate bundle cannot be less than 1 . This property of candidate bundles is useful in proof of lemma 3.6, allowing us to infer all prices from prices on only a few items.

LemMa 3.5. In an equilibrium with prices $\mathbf{p}$, all candidate bundles have price at least 1.

Proof. We prove this by contradiction. Assume that there exists an agent $i$ such that the price of one her candidate bundles $b$ is less than one. Assume that $i$ 's most preferred item is $j$. We have the following cases

- $b=(z) \neq(j)$ : In this case, since $i$ 's most preferred item is unique, there exists an $\epsilon>0$ such that if $i$ gets $1-\epsilon$ unit of item $z$ and $\epsilon$ unit of item $j$, her total price is still less than 1, but her utility is more than $v_{i z}$. This is a contradiction since by the definition of candidate bundles, $i$ 's utility in equilibrium cannot be more than $v_{i z}$.

- $b=(j)$ : In this case, we claim that $\mathbf{x}$ does not allocate any item to $i$ other than $j$. Assume this is not true. If $i$ trades whatever she gets in the equilibrium with $j$, her price will be less than 1 , and since the maximum value for $i$ is unique, her utility will increase. So it should be the case that $i$ does not spend all her money and market will not be cleared. This is a contradiction with the assumption that the prices are market clearing.

- $b=(k, z)$ : Since the maximum value item of $i$ is unique, her value $b$ should be less than $v_{i j}$. Now since the unit price of $b$ is less than one, there exists $\epsilon>0$ such that $\epsilon p_{j}+(1-\epsilon) p_{b}<1$. However, if $i$ gets $\epsilon$ from item $j$ and $1-\epsilon$ from bundle $b$, his utility is more than $v_{i b}$. This is a contradiction with the definition of candidate bundles.

So all the possible cases reach a contradiction and we are done.

Next we wish to find the prices for all items. We will show that if one knows for each agent her utility, the price of only one item in her optimum bundles, and we use the set of candidate bundles defined above, we can find a the price of all the items which are in one of her optimum bundles.

LEMma 3.6. Consider an equilibrium where we know for each agent $i$, the utility $u_{i}$ of the agent, and the price of a single item $j$ which is in a bundle of $B_{i}$ with two items. Using these values, and the notion of candidate bundles defined above, we can find the price of all items in polynomial time.

Proof. The key for finding the prices is the observation that if for a bundle $b=(j, k)$, we have $\alpha_{b}$ and $p_{j}$, then we can find $p_{k}$. This fact, combined with lemma 3.4, imply that if for each agent $i$, we know the price of one item in one of her optimum bundles, then we can find the price of all the items in her optimum bundles. The only problem is that we do not know which one of her candidate bundles is also one of her optimum bundles.

Assume that for each agent $i$, we know a good $g_{i} \in[\mathrm{m}]$ is in one of her optimum bundles and we have a variable for $g_{i}$ 's price. To find a formula for price of other items with the variables we have defined so far, consider the following 
game. Assume that $i$ is playing a game, in which she wants to maximize the number of her optimum bundles. She can participate in this game by proposing a price for all the items in her candidate bundles, knowing $p_{g_{i}}$ and her candidate bundles. W.l.o.g assume that $p_{g_{i}}<0$. For each of her candidate bundles $b=\left(g_{i}, j\right), i$ first proposes price $p_{j}=\frac{1-\alpha_{b} g_{i}}{1-\alpha_{b}}$ for item $j$, then for each of her candidate bundles $b^{\prime}=(k, j)$, she proposes price $p_{k}=\frac{1-\left(1-\alpha_{b^{\prime}}\right) p_{j}}{\alpha_{b^{\prime}}}$ for item $k$. Finally she proposes price 1 for all the items which form a bundle alone. By doing this, if any of her proposed prices is chosen, that item will be in her optimum bundles. Now, for each item $j$, we, the game coordinator, choose the maximum price for $j$ among all the prices which were proposed by the agents for $j$ and set that to be the price of item $j$.

Note that in equilibrium, we have to choose the maximum proposed price, since if we choose less than that, the agents with higher proposals will have candidate bundles whose price is less than 1, this is a contradiction with lemma 3.5.

In order to use the above lemma, we should be able to do two things: (i) For each agent $i$, select an item $j$ that is in one of $i$ 's optimum bundle with two items (if such an item exists), and set its price $p_{j}$. (ii) For each item $j$, find the maximum proposed price among all the proposed prices for that item, with proposed price of 1 of items in single item candidate bundles for any agent (including agents with no special item). We can do the first task by checking all possible assignments of the special items with defining $O\left(\mathrm{~m}^{n}\right)$ separate equilibrium structures for each possible selection of one special item assigned to each agent, and checking them separately. Since the number of agents is constant, the number of different equilibrium structures is polynomial. For each case, we use $O(n)$ variables, one for agent utility, and one for the price of the proposed special items for agents. To define prices of other items, we use candidate bundles for each agent to define candidate prices, add polynomial comparing the expressions for candidate prices. The actual price of the item is the highest of all prices as shown in the proof of Lemma 3.6, which is now set uniquely in each cell. Note that if an agent $i$ is proposing a price for an item $j$ which is higher than the proposed price of another agent $i^{\prime}$ and $j$ is the special item of $i^{\prime}$, then this cell cannot contain equilibria.

Lemma 3.7. Consider the space of at most $2 n$ variables including agents' utilities and price of one item in each agent's optimum bundles. Now we add $O\left(\mathrm{mn}^{2}\right)$ polynomials: comparing utilities to item values, and comparing candidate item prices using candidate budges, as defined in Lemma 3.6, the sign of these polynomials gives us a formula for the price of each item as well as the set of optimum bundles of each agent.

\subsection{Characterizing the Equilibria}

In this section, we add a set of new variables and polynomials to the set of variables and polynomials defined in Section 3.1, in order to determine whether each cell contains equilibria. The new variables will help us define assignments. We cannot directly define a variable representing the allocation of all goods/bundles to agents, since the number of these is not constant.

The key idea is to show that for every equilibrium pricing, there is a specific allocation of items to agents where the number of items which is being shared between multiple agents is very small, and the allocation has a special structure. This helps us to significantly reduce the number of variables needed to define allocations. Consider two agents $i<j$, and all items of price $p_{k}<1$ in sorted by price as shown by Figure 1 . We will show that there is an equilibrium allocation with only two of these items shared between $i$ and $j$, and the structure indicated by the figure, and the analogous structure for items of price $p_{k}>1$. If we know the shared items, this structure helps us with finding the owner of the items which only have a single owner in the allocation, hence finding the allocation of each agent using allocation variables only for shared items. Before stating the properties of this allocation in Lemma 3.8, we have to define some notations. 


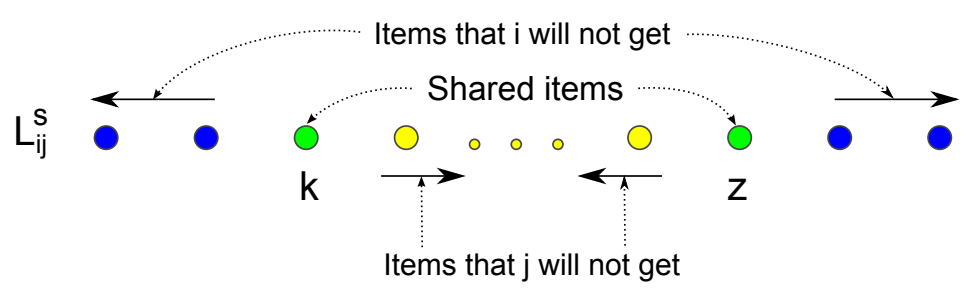

Fig. 1. The nodes are the items in $S$ (one side of $G$ ) that are in a optimum bundle of both $i$ and $j(i<j$ ), which are sorted by their prices. The figure shows the items that $i$ and $j$ share (green nodes), the items that of $i$ or $j$ will not get in the special allocation (blue and yellow nodes).

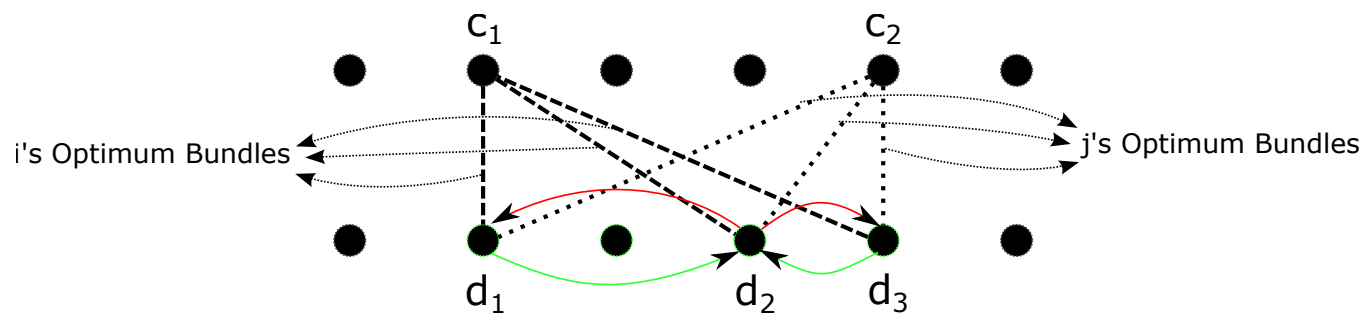

Fig. 2. The arrows show the trades of items between agents $i$ and $j$ in the proof of Lemma 3.8.

Consider a bipartite graph $G=(S, T, E)$ in which vertices in $S$ are the items with price less than 1 and vertices in $T$ are the items with price more than 1. For simplicity, we sort items in each side of $G$ by their price and break ties with items' indexes. Figure 1 is one side of this bipartite graph. For each bundle, we put an edge in the graph which connects the two items in the bundle. For every pair of agents $i$ and $j$, let $B_{i j}^{S}=S \cap\left(V\left(B_{i}\right) \cap V\left(B_{j}\right)\right)$ and $B_{i j}^{T}=T \cap\left(V\left(B_{i}\right) \cap V\left(B_{j}\right)\right)$. Furthermore, let $H$ be the set of items whose price is 1 and let $B_{i}^{H}$ be the optimum bundles of agent $i$ which has exactly 1 item.

For each pair of agents $i$ and $j$, let $L_{i j}^{S}$ and $L_{i j}^{T}$ be the list of items in $B_{i j}^{S}$ and $B_{i j}^{T}$ sorted by their price in increasing order (break ties with index of the items), respectively, the set of items of price above 1 (and below 1) that are part of optimum bundles for both $i$ and $j$. Figure 2 is showing a part of the above bipartite graph for a pair of agents.

The following Lemma claims that there is an equilibrium allocation of a special structure suggested by Figure 1: on each side of the bipartite graph the agents share at most two items, and the agent with lower index only gets items between the two shared items, while the agent with higher index only gets items outside this interval as shown by Figure 1.

The main idea of the proof is that given any equilibrium allocation, we can make each pair of agents that violate the properties trade their items, as illustrated by the Figure 2. Note that the running time of this trading process is not important, since we only use it to show that for any equilibrium pricing an allocation with the desired structure exists.

LEMMA 3.8. For every equilibrium pricing $\mathbf{p}$, there exists an equilibrium allocation $\mathbf{x}$ of items to agents such that for every pair of agents $i$ and $j(1 \leq i<j \leq n)$

1. There are at most 2 items that $X$ is allocating to both $i$ and $j$ on each side of $G$. Furthermore, if $k$ and $z$ are two items in $S$ (T) such that $p_{k} \leq p_{z}$ and $i$ and $j$ are sharing $k$ and $z$, $i$ only gets items between $k$ and $z$ in the order sorted by price, while $j$ does not get any of the items from $B_{i j}^{S}\left(B_{i j}^{T}\right)$ whose whose position in $L_{i j}^{S}\left(L_{i j}^{T}\right)$ is between $k$ and $z$ in the order (see Figure 1). 


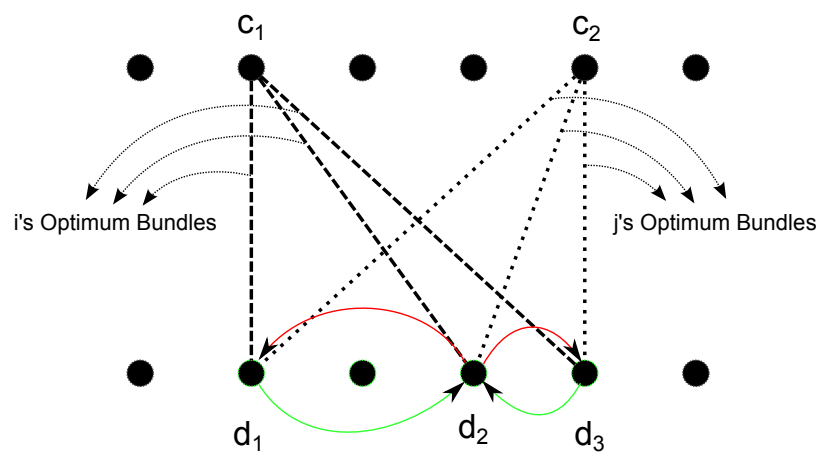

Fig. 3. Red and green arrows shows the trades of items between agent $i$ and $j$ in proof of lemma 3.8 .

2. There is at most one item $k$ in $B_{i}^{H} \cap B_{j}^{H}$ that is shared by $i$ and $j$ in $\mathbf{x}$, and $i$ only get items from $B_{i}^{H}$ whose index is lower than $k$ and $j$ only gets items whose index is higher than $k$.

Proof. Suppose that we have an allocation of bundles to agents $y$ in an equilibrium. We want to reallocate these bundles so that it satisfies the conditions of the lemma.

Assume that there exist two agents $1 \leq i<j \leq n$, such that there are tree bundles $a_{1}=\left(c_{1}, d_{1}\right), a_{2}=\left(c_{1}, d_{2}\right)$ and $a_{3}=\left(c_{1}, d_{3}\right)$ such that $a_{1}, a_{2}, a_{3} \in B_{i}$ and three bundles $b_{1}=\left(c_{2}, d_{1}\right), b_{2}=\left(c_{2}, d_{2}\right)$ and $b_{3}=\left(c_{2}, d_{3}\right)$ such that $b_{1}, b_{2}, b_{3} \in B_{j}$. Suppose that $p_{d_{1}} \leq p_{d_{2}} \leq p_{d_{3}}$ and $Y \mathbf{y}$ is allocating $y_{i d_{1}}, y_{i d_{3}}>0$ of $a_{1}$ and $a_{3}$ to agent $i$, and $y_{j d_{2}}>0$ of $d_{2}$ to agent $j$. Furthermore, assume that $y_{i c_{1}}, y_{j c_{2}}>0$.

Since $p_{d_{1}} \leq p_{d_{2}} \leq p_{d_{3}}$, there is $0<\beta<1$ such that $\beta p_{d_{1}}+(1-\beta) p_{d_{3}}=p_{d_{2}}$. So if we remove $\beta z$ from $y_{i d_{1}}$ and $(1-\beta) z$ from $y_{i d_{3}}$ and add $z$ to $y_{i d_{2}}$ then the total cost of agent $i$ will be the same. Similarly, if we add $\beta z$ to $y_{j d_{1}}$ and $(1-\beta) z$ to $y_{j d_{3}}$ and remove $z$ from $y_{j d_{2}}$, then the total cost of $j$ will not change. Furthermore, it is easy to see that doing this does not affect the matching constraints. Figure 3 demonstrates this procedure.

Now, we have to show that doing this does not change the utility of $i$ and $j$. From the assumption that $Y y$ is an equilibrium allocation, it follows that by doing this, the utility of $i$ and $j$ cannot increase. Assume that by doing this utility of agent $i$ decreases. We have

$$
\begin{aligned}
u_{i} & =\alpha_{a_{2}} v_{i d_{2}}+\left(1-\alpha_{a_{2}}\right) v_{i c_{1}} \\
& <\alpha_{a_{2}}\left(\beta v_{i d_{1}}+(1-\beta) v_{i d_{3}}\right)+\left(1-\alpha_{a_{2}}\right) v_{i c_{1}} \\
& =\beta \alpha_{a_{2}} v_{i d_{1}}+(1-\beta) \alpha_{a_{2}} v_{i d_{3}}+\left(1-\alpha_{a_{2}}\right) v_{i c_{1}}
\end{aligned}
$$

similarly we have

$$
\beta \alpha_{a_{2}} p_{d_{1}}+(1-\beta) \alpha_{a_{2}} p_{d_{3}}+\left(1-\alpha_{a_{2}}\right) p_{c_{1}}=1
$$

This means that if agent $i$ only use these three items with these ratios, he gets one unit of item, spends at most 1 unit of money and her utility will be more than $u_{i}$. This is a contradiction. The argument for agent $j$ is similar to the argument for agent $i$. 
For each pair of agents $1 \leq i<j \leq n$, we find the cheapest item $k$ and the most expensive item $z$ in $S$ that $i$ owns, if there is an item $r$ in between $k$ and $z$ in the ordered list of items in $S$ that $j$ owns, we switch the allocation we switch the ownership of these items until, one of the following cases happen

- $j$ runs out of item $r$.

- $i$ runs out of item $k$.

- $i$ runs out of item $z$

Let $\Phi$ be a potential function which is equal to $|S|$ minus the the position of highest positioned item that $i$ owns in the ordered list, plus the position of the lowest positioned item that $i$ owns in the ordered list, plus the number of items for which $j$ is a shareholder and are between the the two in the ordered list. If we repeat the above procedure, each time this potential function will decrease by 1 . Since the potential function is always non-negative, we cannot continue the above procedure forever. This means that at after some iterations, we will reach an equilibrium allocation which satisfies the first condition for this pair of agents.

We repeat this procedure until such pair of agents and set of items with these properties do not exist. We also do the same to the allocation of items on the other side of $G(T)$ to agents. For the items in $B_{i}^{H} \cap B_{j}^{H}$, one can also transfer items between $i$ and $j$ to satisfy the second condition by finding two items that violate the condition and switch their ownership. The procedure defined for satisfying these conditions are an easier version of the previous procedure.

From now on, we focus on finding and characterizing the specific equilibrium allocation which is guaranteed to exist by this lemma. By using Lemma 3.8, for every pair of agents $i, j \in[n]$ we can use 5 item indexes $f_{i j}^{S}, r_{i j}^{S}, f_{i j}^{T}, r_{i j}^{T}$, and $h_{i j}$ that tell us which items are shared by agent $i$ and $j$. We can assume that we know what are these shared items by simply checking all the possible $O\left(m^{5 n^{2}}\right)$ of these combinations. At the start of the algorithm, we fix the 5 shared items for each pair of agents and an item in optimum bundle of each agent (which we discussed in Section 3.1). We call this set of items associated with each agent and pairs of agents the structure of the equilibrium. For each such a structure, we will aim to decide if there is an equilibrium with the given structure.

When considering equilibria of a given structure, we define variables for the allocation of the at most $5 n^{2}$ shared items, but do not define variable for allocations of other items. Next we show that given the allocations of the shared items, we can (i) infer allocations of all other items using the structure of Lemma 3.8, and (ii) can also define polynomials whose sign will tell us if there is an equilibrium with the given structure and allocation of shared items.

Since all the items should be sold in the equilibrium, for each agent $i$, all the items that are in only in $i$ 's optimum bundles, should get allocated to $i$. Second, we have defined a variable that indicates the share of each agent for the shared items. The only thing left is to consider items that are in the set of the optimum bundles of multiple agents, but are not shared by these agents.

Lemma 3.8 helps us find allocation of this set of items. We start with the agent with the lowest index (agent 1) and one side of $G$, say $S$. The items that agent 1 gets, are the ones that satisfy all the constraints given to us by second part of the lemma. In order to check whether for an item $j$ all these constraints hold, for each agent $i$, we can look at the position of item $j$ in $L_{1 i}^{S}$. If $j$ 's position is between position of $f_{1 i}^{S}$ and $r_{1 i}^{S}$ in the list, for all the choices of $i>1$, then it satisfies all the constraints. By lemma 3.8, we know this is a necessary and sufficient condition for $j$ to get allocated to agent 1 . We do this for $S$ and $T$ separately, and remove the items that get allocated to agent 1 . Now, we repeat this procedure for agent 2 , but only check the constraints for agents $i>2$, then remove the items that agent 2 gets. We repeat this for rest of agents based on their indexes. We can do the same procedure for items in $H$ to find which agent 
is getting what item. Now, we are ready to exactly specify what are the necessary and sufficient conditions for the prices in each cell to be the equilibrium prices. This process is summarized in the following lemma.

LEMma 3.9. Consider a structure of special items for agents, and shared items between agents (as defined after Lemma 3.8). Now consider a cell in the space of variables of agent utilities, prices of the special items, and allocation shares of the shared items, defined by the polynomials that help define prices of all items. The prices and allocation of this cell forms an equilibrium, if and only if the allocation defined above satisfies the following constraints

1. All the items get fully allocated to agents.

2. Each agent gets exactly one unit of items.

3. For each agent $i$, the total cost of buying the items allocated to $i$ is exactly 1 .

4. For each agent $i$, each of the items allocated to $i$ is in one of her optimum bundles.

Proof. $\Rightarrow$ The first, second and third condition follow from the market clearing conditions. The fourth condition directly follows from part c of lemma 3.2.

$\Leftarrow$ From the first and second condition, we know that the pricing and allocation are market clearing. From the second, third and fourth condition, and the argument in proof of part c of lemma 3.2 we know that the allocation of items to each agent can be rewritten as her optimum bundles to her. So, from the definition of optimum bundles we know that the allocation of items to agents is optimal. Therefore, the prices and allocation are in equilibrium state.

The final thing we need to do is to define a set of polynomials for checking the above conditions. The first conditions holds for an item $j$, if summing up the share of each agent for that item, the sum is equal to $C_{j}$. This can be handled by adding one polynomial for each item $j$.

The second condition holds for an agent $i$, if when we sum all the items (including the proportion of the shared items) that $i$ gets, this sum is exactly 1 . So we can also check this condition by adding a polynomial for each agent. Note that we can do this since we explained how to find out what is the allocation of items to agents for this cell.

The third condition holds by Lemma 3.9 if multiplying the share of each agent for an item by its price and summing over all items, we get 1 for all the agents. We can do this by defining a polynomial for each agent and checking its sign. The fourth condition is guaranteed to hold by definition. The following theorem summarizes how we equilibria are cells of the constraints discussed throughout this section.

THEOREM 3.10. Consider an equilibrium structure, and the space of the $O(n)$ variables for agents' utilities and price of one special item in each agent's optimum bundle, the $O\left(n^{2}\right)$ variables for allocation of shared items between the agent. Divide this space into cells by the signs of the polynomials defined in the previous section, along with the $O\left(m^{2}+n\right)$ polynomials defined just above, for checking the existence of the special equilibrium allocation. The sign of these polynomials fully determines either that the vectors in this cell can be extended to form equilibria.

Using this structure Theorem allows us to prove Theorem 3.1.

Proof of Theorem 3.1. We use theorem 2.1 as the base of our algorithm. We start by fixing the structure of the equilibrium, selecting a single item from the optimum bundles of each agent, and selecting fixing 5 items shared for every pair of agents. We check all the possible combinations, at most $O\left(m^{n+5 n^{2}}\right)$ options, which is polynomial in $m$ for fixed $n$.

For a given equilibrium structure, we use the $O(n)$ variables, the agent utilities, and $O(m n)$ polynomials defined in section 3.1 to find a set of candidate bundles for each agent. Then we use an additional $O(n)$ variables, the prices of the special items for each agent, and $O\left(m n^{2}\right)$ polynomials in order to find a formula for the prices and the set of optimum 
bundles of each agent in each cell. Finally, we use the last set of $O\left(n^{2}\right)$ variables, the assignments of shared items, and $O\left(m^{2}+n\right)$ additional polynomials, in order to check whether the set of prices in the feasible cell are equilibrium prices with the given structure. The degree of the defined polynomials is polynomial. We check all the non-empty cells of the resulting system, taking time polynomial on $m$ for any fixed $n$ by Theorem 2.1. Since the equilibrium exists, it should be in one of the non-empty cells.

Finally, if the prices of the cell are equilibrium prices, we take any vector from that cell, and extend it to get an equilibrium pricing and allocation. After we have the equilibrium prices $\mathbf{p}$, we can also find each agent's allocation by finding a solution of the following set of inequalities.

$$
\begin{array}{lr}
\sum_{b \in B_{i}} x_{i b}=1 & \forall i \in[n] \\
\sum_{i}\left(\sum_{b: b=(j, k)} \alpha_{b} x_{i b}+\sum_{b: b=(k, j)}\left(1-\alpha_{b}\right) x_{i b}+\sum_{b: b=(j)} x_{i b}\right)=C_{j} & \forall j \in[m] \\
x_{i b} \geq 0 & \forall i \in[n], b \in B
\end{array}
$$

In which, $x_{b i}$ is the amount of bundle $b$ used by agent $i$. Note that since we know this bundling is associated with an equilibrium, the feasible region of the above inequalities is not empty. Finally, the allocation of each agent $i$ for item $j$ in this equilibrium is

$$
x_{i j}=\sum_{b: b=(j, k)} \alpha_{b} x_{i b}+\sum_{b: b=(k, j)}\left(1-\alpha_{b}\right) x_{i b}+\sum_{b: b=(j)} x_{i b}
$$

\subsection{Relaxing Full Budget Spent Assumption of Agents in Equilibria}

So far, we have assumed each agent fully spend her budget and introduced an algorithm for finding an equilibrium with this assumption. While equilibria where all agents spend their budget do exists (as shown in Appendix $\mathrm{C}$ of the full version of this paper [3]), there may be equilibria where not all agents spend their budgets. In this section, we describe how the same algorithm can be modified to find an equilibrium which does not require this assumption. The following lemma shows that if an agent does not fully spend her budget in an equilibrium, she will get exactly one unit of her most desired item, hence she cannot get any part of the other items. Recall that we assume that for each agent, her most preferred item is unique.

LeмmA 3.11. In an equilibrium, with price vector $\mathbf{p}$ and equilibrium allocation $\mathbf{x}$, for each agent $i$ whose most preferred item is $j$, if $i$ does not fully spend her one unit of money then $x_{i j}=1$.

Proof. We use contradiction. Assume $i$ is not fully spend her budget in an equilibrium, but there exists an item $z$ such that $x_{i z}>0$ and $z$ is not her most preferred item. Let $b_{i}^{\prime}$ be $i$ 's leftover money and $j$ be her most preferred item. If $p_{z} \geq p_{j}$ then $i$ can trade her share of item $z$ for the same amount of item $j$ and increase her utility, which contradicts the equilibrium condition. If $p_{z}<p_{j}$ then, since $i$ has $b_{i}^{\prime}$ unit of extra money, $i$ can afford to trade $\min \left(\frac{p_{j}-p_{z}}{b_{i}^{\prime}}, x_{i z}\right)>0$ unit of item $z$ with the same amount of item $j$ and increase her utility. This contradicts the fact that in equilibrium, utility of each agent should be optimum and we are done.

Since the number of agents is fixed, we can iterate over all the $2^{n}$ settings each of which tells us whether each agent fully spend her budget or not. In each setting, before we start the algorithm, for each agent that does not fully spent 
her budget, we remove one unit from her most preferred item. From lemma 3.11 we know that if this setting leads to an equilibrium, this will be the only valid allocation of items to these agents. We also remove these agents themselves and continue the algorithm assuming all the remaining agents fully spend their budget as before. If in a setting, enough of the most preferred items of the agents who do not fully spend their budget is not available, then it simply means that the setting cannot lead to an equilibrium, so we ignore the setting and iterate to the next one.

Note that if an agent is not fully spending her budget, from lemma 3.11 we know that the price of her most preferred item should be at most 1 . Therefore, in our algorithm after formulating the prices in the algorithm (described in section 3.1), for each item that was partially allocated to the agents who do not fully spent their budget, we add a constraint that its price should be at most 1 . Then we continue with the algorithm to find the equilibrium given the initial setting. Because such equilibrium always exists, at least one of the initial settings will lead to finding an equilibrium price vector and allocation.

\section{ACKNOWLEDGMENTS}

We want to thank the generous support of NSF via grant CCF-1563714, ONR via grant N00014-08-1-0031, and a Google Research Grant.

\section{REFERENCES}

[1] Atila Abdulkadiroglu and Tayfun Sonmez. 1998. Random Serial Dictatorship and the Core from Random Endowments in House Allocation Problems. Econometrica 66, 3 (May 1998), 689-702. https:/ideas.repec.org/a/ecm/emetrp/v66y1998i3p689-702.html

[2] Marek Adamczyk, Piotr Sankowski, and Qiang Zhang. 2014. Efficiency of Truthful and Symmetric Mechanisms in One-Sided Matching. In Algorithmic Game Theory - 7th International Symposium, SAGT 2014, Haifa, Israel, September 30 - October 2, 2014. Proceedings. $13-24$.

[3] Saeed Alaei, Pooya Jalaly, and Éva Tardos. 2017. Computing Equilibrium in Matching Markets. CoRR abs/1703.10689 (2017). http://arxiv.org/abs/ 1703.10689

[4] S. Basu, R. Pollack, and M.-F. Roy. 1998. A new algorithm to find a point in every cell defined by a family of polynomials. In Quantifier Elimination and Cylindrical Algebraic Decomposition (Texts and Mongraphs in Symbolic Computation), B. Caviness and J. Johnson (Eds.). Springer-Verlag, 341-350.

[5] Anand Bhalgat, Deeparnab Chakrabarty, and Sanjeev Khanna. 2011. Social Welfare in One-Sided Matching Markets without Money. In Approximation, Randomization, and Combinatorial Optimization. Algorithms and Techniques - 14th International Workshop, APPROX 2011, and 15th International Workshop, RANDOM 2011, Princeton, Nf, USA, August 17-19, 2011. Proceedings. 87-98.

[6] Anna Bogomolnaia and Herve Moulin. 2001. A New Solution to the Random Assignment Problem. Fournal of Economic Theory 100, 2 (2001), 295 328. DOI : http://dx.doi.org/10.1006/jeth.2000.2710

[7] Bruno Codenotti and Kasturi Varadarajan. 2007. Computation of market equilibria by convex programming. In Algorithmic Game Theory, Noam Nisan, Tim Roughgarden, Eva Tardos, and Vijay V. Vazirani (Eds.). Cambridge University Press, Chapter 6, $135-158$.

[8] Nikhil R. Devanur and Ravi Kannan. 2008. Market Equilibria in Polynomial Time for Fixed Number of Goods or Agents. In Proceedings of the 2008 49th Annual IEEE Symposium on Foundations of Computer Science (FOCS '08). IEEE Computer Society, Washington, DC, USA, 45-53. D0I : http://dx.doi.org/10.1109/FOCS.2008.30

[9] Danny Dolev, Dror G. Feitelson, Joseph Y. Halpern, Raz Kupferman, and Nathan Linial. 2012. No Justified Complaints: On Fair Sharing of Multiple Resources. In Proceedings of the 3rd Innovations in Theoretical Computer Science Conference (ITCS '12). ACM, New York, NY, USA, 68-75. DOI : http://dx.doi.org/10.1145/2090236.2090243

[10] Federico Echenique and Adam Wierman. 2012. Finding a walrasian equilibrium is easy for a fixed number of agents.. In EC. 495.

[11] Avital Gutman and Noam Nisan. 2012. Fair Allocation Without Trade. In Proceedings of the 11th International Conference on Autonomous Agents and Multiagent Systems - Volume 2 (AAMAS '12). International Foundation for Autonomous Agents and Multiagent Systems, Richland, SC, 719-728. http://dl.acm.org/citation.cfm?id=2343776.2343799

[12] Aanund Hylland and Richard Zeckhauser. 1979. The Efficient Allocation of Individuals to Positions. Fournal of Political Economy 87, 2 (April 1979), 293-314. https://ideas.repec.org/a/ucp/jpolec/v87y1979i2p293-314.html

[13] Xiaodong Wang and José F. Martínez. 2015. XChange: A market-based approach to scalable dynamic multi-resource allocation in multicore architectures. In International Symposium on High-Performance Computer Architecture (HPCA). Bay Area, CA.

[14] Lin Zhou. 1990. On a conjecture by gale about one-sided matching problems. fournal of Economic Theory 52, 1 (1990), 123-135. http://EconPapers. repec.org/RePEc:eee:jetheo:v:52:y:1990:i:1:p:123-135 